\title{
Long Noncoding RNA FOXD2-AS1 Promotes Pancreas Adenocarcinoma Cell Invasion and Migration by Sponging miR-30a-3p to Upregulate COX-2
}

\author{
Zhihua Ye, Yingyu Yang, Ying Wei, Lamei Li, Xinyi Wang, \& Junkai Zhang* \\ Department of Medical Oncology Center, Zhongshan People's Hospital, Zhongshan City, Guangdong Province, \\ 528403, P.R. China \\ *Address all correspondence to: Junkai Zhang, Department of Oncology, Department of Medical Oncology Center, Zhongshan People's Hospital, \\ Zhongshan City, Guangdong Province, 528403, P.R. China; Tel.: +0760-88823566, E-mail: ij6029@163.com
}

\begin{abstract}
Introduction: FOXD2-AS1 is known to promote the development of several cancers. However, its role in pancreatic adenocarcinoma (PAAD) is unclear.

Methods: Expression of FOXD2-AS1 and miR-30a-3p in PAAD patients was analyzed with RT-qPCR. A follow-up study was performed to analyze the prognostic value of FOXD2-AS1 for PAAD. Overexpression assays were performed to analyze the crosstalk between FOXD2-AS1 and miR-30a-3p. Cell invasion and migration were analyzed by transwell assays.
\end{abstract}

\begin{abstract}
Results: Analysis of the TCGA dataset revealed that FOXD2-AS1 was upregulated in PAAD tissues compared to the non-cancer tissues (1.89 vs. 0.2 TPM), indicating potential involvement of FOXD2-AS1 in PAAD. Our own data also showed FOXD2-AS1 was overexpressed in PAAD. Moreover, high FOXD2-AS1 levels predicted poor survival. It is predicted that miR-30a-3p can bind FOXD2-AS1, while their overexpression did not affect each other's expression. Correlation analysis revealed a significant correlation between FOXD2-AS1 and COX-2. In addition, FOXD2-AS1 overexpression increased COX-2 level, while miR-30a-3p played an opposite role. FOXD2-AS1 and COX-2 overexpression increased PAAD cell invasion and migration. MiR-30a-3p played an opposite role and inhibited the effects of FOXD2-AS1 and COX-2 overexpression.
\end{abstract}

Conclusion: FOXD2-AS1 may promote PAAD cell invasion and migration by sponging miR-30a-3p to upregulate COX-2.

KEY WORDS: FOXD2-AS1, pancreatic adenocarcinoma, COX-2, miR-30a-3p

\section{INTRODUCTION}

Mortality of pancreatic cancer (PC) ranks seventh place among all cancers. ${ }^{1}$ In many countries, $\mathrm{PC}$ is predicted to surpass breast cancer as the third major cause of cancer mortalities in the near future. ${ }^{1}$ In 2018, PC occurred in 458,918 patients, accounting for $2.5 \%$ of all new cancer cases. In 2018, PAAD caused 432,242 deaths, accounting for $4.5 \%$ of all cancer deaths. ${ }^{2}$ Pancreatic adenocarcinoma (PAAD) is the major subtype of $\mathrm{PC}$ and accounts for $85 \%$ of all PC cases. ${ }^{3}$ Due to the lack of effective therapies and the extremely aggressive nature, less than $5 \%$ of PAAD patients survive more than 5 years. ${ }^{4}$ However, the molecular pathogenesis of PAAD is unclear, and the development of targeted therapies is limited. ${ }^{5}$
Noncoding RNAs (ncRNAs) are critical players in diverse pathological and physiological processes.${ }^{67}$ Despite the lack of protein-coding capacity, ncRNAs (ncRNAs), such as long ncRNAs (lncRNAs) and miRNAs, play their roles by regulating downstream gene expression. ${ }^{8,9}$ Moreover, it has been established that lncRNAs may serve as the endogenous competing RNAs for miRNAs to suppress the function of miRNAs without affecting their expression. ${ }^{8,9}$ Therefore, exploring the interaction between lncRNAs and miRNAs may provide novel insights into the development of anti-cancer approaches. However, the function of most miRNAs and lncRNAs in cancer biology is unclear. LncRNA FOXD2-AS1 is an oncogenic lncRNA in many cancers, such as liver cancer and lung cancer. ${ }^{10,11}$ However, its function in PAAD is unknown. We analyzed 
the TCGA dataset and observed that FOXD2-AS1 was upregulated in PAAD tissues compared to non-cancer tissues ( 1.89 vs. 0.2 TPM), indicating its involvement in this disease. Bioinformatics analysis showed that FOXD2-AS1 could interact with miR30a-3p, which plays a tumor-suppressive role in gastric cancer by targeting COX-2. ${ }^{12}$ We then speculated that FOXD2-AS1 could directly interact with miR-30a-3p to indirectly affect COX-2 expression, thereby participating in PAAD. This study was therefore carried out to analyze the roles of FOXD2-AS1, miR-30a-3p, and COX-2 in PAAD.

\section{MATERIALS AND METHODS}

\section{A. PAAD Patients}

Fifty-four (33 males and 21 females, aged 38 to 67 years, $52.1 \pm 6.2$ years) out of the 98 PAAD patients admitted to Zhongshan People's Hospital between May 2011 and May 2014 were enrolled in this study. Ethics approval was obtained from the hospital (approval number: RE\&516). Inclusion criteria were as follows: (1) confirmed by histopathological test, (2) newly diagnosed cases, and (3) completed treatment and 5-year follow-up. Exclusion criteria were as follows: (1) recurrent PAAD, (2) multiple complications were diagnosed, and (3) therapies were initiated before this study. All patients were informed of the experiments and signed the informed consent.

\section{B. Treatment and Follow-Up}

According to the AJCC criteria, the 54 enrolled patients included 17, 18, and 19 cases at clinical stages II, III, and IV, respectively. Treatment strategies, such as surgical resection combined with chemotherapy or chemotherapy alone were determined mainly based on cancer stages. From the day of admission, follow-up was performed for 5 years to record deaths. All patients completed the follow-up.

\section{Biopsies and PAAD Cells}

Before the initiation of therapies, a biopsy was performed to collect PAAD and adjacent (within $3 \mathrm{~cm}$ around tumors) non-cancer tissues. All tissue samples were confirmed by histopathological exams.

Human PAAD cell line Capan-2 (ATCC, Manassas, VA, USA) was used as a PAAD cell model and cultured in a mixture containing $10 \%$ FBS and $90 \%$ McCoy's 5a Medium under the conditions of $5 \%$ $\mathrm{CO}_{2}, 37^{\circ} \mathrm{C}$, and $95 \%$ humidity.

\section{Constructs, miRNA, and Transfections}

PcDNA3.1 vector was used to construct vectors expressing FOXD2-AS1 and COX-2. Negative control (NC) miRNA and miR-30a-3p mimic were from Sigma-Aldrich (St. Louis, MO, USA). At the confluence of $70-80 \%$, Capan-2 cells were harvested, and $10 \mathrm{nM}$ vectors (empty pcDNA3.1 vector as $\mathrm{NC}$ group) or $40 \mathrm{nM}$ miRNAs (NC miRNA as $\mathrm{NC}$ group) were transfected into $10^{6}$ Capan-2 cells through transient transfections mediated by Lipofectamine 2000 (Sigma-Aldrich). Subsequent experiments were carried out $24 \mathrm{~h}$ later. Control (C) cells were untransfected cells.

\section{E. RNA Extractions and qPCR}

Biopsies were ground in liquid nitrogen, and $\mathrm{Ca}-$ pan-2 cells $\left(10^{5}\right)$ were harvested at $24 \mathrm{~h}$ post-transfection. Total RNAs were extracted from the biopsies and cells using RNAzol reagent (Sigma-Aldrich) following the methods described in the instructions from the manufacturer. RNAs were precipitated using $85 \%$ ethanol. By doing this, miRNAs were also precipitated. DNase I was used to digest total RNA samples for $2 \mathrm{~h}$ at $37^{\circ} \mathrm{C}$ to remove gDNAs. Power cDNA Synthesis Kit (iNtRON Biotechnology, South Korea) was used to prepare cDNA samples through reverse transcriptions, and all qPCR assays were performed using KAPA SYBR FAST qPCR Master Mix (Kapa Biosystems, Wilmington, MA, USA). Expression levels of FOXD2-AS1 and COX-2 mRNA were normalized to GAPDH, $18 \mathrm{~S}$ rRNA, and $\beta$-actin. It is worth noting that similar results were obtained from different internal controls. To detect the levels of mature miR-30a-3p expression, all steps were completed using All-in-One ${ }^{\mathrm{TM}}$ miRNA qRT-PCR Detection Kit (GeneCopoeia, Rockville, 
MD, USA). Expression levels of miR-30a-3p were normalized to U6. All experiments were repeated 3 times, and all data were processed using the $2^{-\Delta \Delta \mathrm{Ct}}$ method. ${ }^{13}$

\section{F. Western Blot}

Proteins were extracted using RIPA solution (Sigma-Aldrich) and quantified using BCA assay (Sangon Biotech, Shanghai, China). After denaturation in boiling water for $10 \mathrm{~min}, 10 \mu \mathrm{g}$ proteins were separated on $10 \%$ SDS-PAGE and transferred onto PVDF membranes. The membranes were blocked in 5\% fat-free milk (PBS) and incubated with rabbit anti-GAPDH (1:1700, ab8245; Abcam, Cambridge, UK) and anti-COX-2 (1:1700, ab52237; Abcam) primary antibodies at $4^{\circ} \mathrm{C}$ for $18 \mathrm{~h}$. After that, membranes were incubated with HRP-labeled goat anti-rabbit secondary antibody (IgG, 1:1600, ab205718; Abcam) for $2 \mathrm{~h}$ at room temperature. Signals were produced using ECL (Sigma-Aldrich) and normalized to GAPDH using ImageJ v.146 software.

\section{G. Transwell Assay}

Transwell assays were performed to measure cell migration and invasion ability. In brief, Capan-2 cells $\left(3 \times 10^{4}\right)$ were harvested at $24 \mathrm{~h}$ post-transfection, resuspended in $1 \mathrm{ml}$ serum-free McCoy's 5a Medium to prepare single-cell suspensions, and loaded onto the upper chamber of transwell plates with the lower chamber filled with a mixture of $20 \%$ FBS and $80 \%$ McCoy's 5a Medium. For invasion assay, the upper chamber membranes were coated with Matrigel (Sigma-Aldrich). After being cultured for $24 \mathrm{~h}$, cells on the lower surface of the membrane were stained with $0.1 \%$ crystal violet (Sigma-Aldrich) and observed under an optical microscope in five visual fields at $40 \times$ magnification to calculate the number of invaded and migrated cells.

\section{H. Statistical Analysis}

Mean values of data from three biological replicates were calculated. Correlations were analyzed by Pearson's correlation coefficient. Differences between PAAD and non-tumor tissues were analyzed by paired $t$-test. ANOVA (one-way) Tukey's test was performed to compare multiple independent groups. Survival analysis was performed through the following steps: (1) grouping the patients into high and low FOXD2-AS1 level groups $(n=27)$ with the median FOXD2-AS1 level in PAAD tissues as the cutoff value, (2) survival curves were plotted based on follow-up data using K-M plotter, and (3) log-rank test was used for comparisons. A $p<0.05$ was statistically significant.

\section{RESULTS}

\section{A. FOXD2-AS1 was Upregulated in PAAD and Predicted Poor Survival}

Analysis of the TCGA dataset was performed using the online program GEPIA (http://gepia.cancer-pku. $\mathrm{cn} /) .{ }^{14}$ The analysis showed that FOXD2-AS1 was upregulated in PAAD tissues compared to non-tumor tissues (1.89 vs. 0.2 TPM), indicating the potential involvement of FOXD2-AS1 in PAAD. We further measured FOXD2-AS1 levels in both PAAD and non-tumor tissues from the 54 patients in this study by qPCR. Paired $t$-test showed that FOXD2-AS1 levels were significantly higher in PAAD tissues than in non-tumor tissues (Fig. 1A, $p<0.05)$. Comparison of survival curves showed that high FOXD2-AS1 level predicted poor survival (Fig. 1B, $p<0.05$ ).

\section{B. MiR-30a-3p Might Bind to FOXD2-AS1 but did not Affect Its Expression}

The interaction between FOXD2-AS1 and miR30a-3p was predicted by IntaRNA. It was observed that miR-30a-3p might be able to bind to FOXD2-AS1 (Fig. 2A). Therefore, we transfected FOXD2-AS1 expression vector and miR-30a-3p mimic into Capan-2 cells to further investigate the interactions between FOXD2-AS1 and miR-30a-3p. Overexpression of FOXD2-AS1 and miR-30a-3p was confirmed by qPCR at $24 \mathrm{~h}$ post-transfection (Fig. 2B, $p<0.05$ ). However, overexpression of 

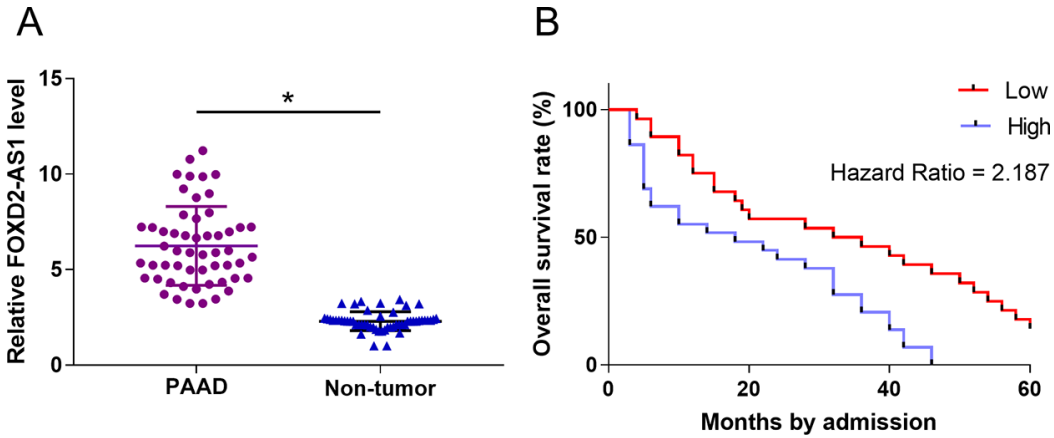

FIG. 1: FOXD2-AS1 was upregulated in PAAD and predicted poor survival. FOXD2-AS1 expression levels in both PAAD and non-tumor tissues from the 54 patients in this study were measured by qPCR and compared using paired t-test (A). ${ }^{*} p<0.05$. Survival curves of high and low FOXD2-AS1 level groups were plotted using K-M plotter and compared by log-rank test (B).

FOXD2-AS1 and miR-30a-3p failed to affect each other's expression (Fig. 2C).

\section{FOXD2-AS1 was Correlated with COX-2 mRNA but not miR-30a-3p in PAAD}

Levels of miR-30a-3p and COX-2 (a target of miR-30a-3p) mRNA were measured by qPCR. The correlations between FOXD2-AS1 and COX-2 mRNA/miR-30a-3p were analyzed by Pearson's correlation coefficient. FOXD2-AS1 levels were significantly and positively correlated with COX-2 mRNA across PAAD samples (Fig. 3A), while the correlation between FOXD2-AS1 and miR-30a-3p across non-tumor samples was not significant (Fig. 3B). COX-2 mRNA levels in PAAD and non-tumor tissues were also compared. The results showed that COX-2 mRNA levels were significantly higher in PAAD tissues than in non-tumor tissues (Fig. 3C, $p<0.05)$.

\section{FOXD2-AS1 Overexpression Upregulated COX-2 via miR-30a-3p}

qPCR and western blot were performed to analyze the effects of FOXD2-AS1 and miR-30a-3p overexpression on COX-2 expression at both mRNA (Fig. 4A) and protein (Fig. 4B) levels. FOXD2-AS1 overexpression upregulated COX-2, while miR$30 a-3 p$ played an opposite role and inhibited the role of FOXD2-AS1 overexpression $(p<0.05)$.
Representative images of western blot were provided in Supplemental Figs. 1 and 2.

\section{E. FOXD2-AS1 Overexpression Promoted Capan-2 Cell Invasion and Migration via miR-30a-3p and COX-2}

Transwell assays were carried out to analyze the effects of transfections on the invasion ability (Fig. $5 \mathrm{~A}$ ) and migration ability (Fig. 5B) of Capan-2 cells. FOXD2-AS1 and COX-2 overexpression increased PAAD cell invasion and migration rates. MiR-30a-3p played an opposite role and inhibited the effects of FOXD2-AS1 and COX-2 overexpression $(p<0.05)$.

\section{DISCUSSION}

We studied the roles of FOXD2-AS1 in PAAD and showed that FOXD2-AS1 was upregulated in PAAD and predicted poor survival. In addition, FOXD2-AS1 might sponge miR-30a-3p to upregulate $\mathrm{COX}-2$.

The oncogenic functions of FOXD2-AS1 have been investigated in many cancers. ${ }^{10,11,15,16}$ FOXD2-AS1 participates in different cancers by interacting with different downstream signaling pathways. For instance, FOXD2-AS1 is upregulated in liver cancer and promotes tumor progression by downregulating CDKN1B (p27) via epigenetic pathways. ${ }^{10}$ FOXD2-AS1 is also highly expressed in lung 
A

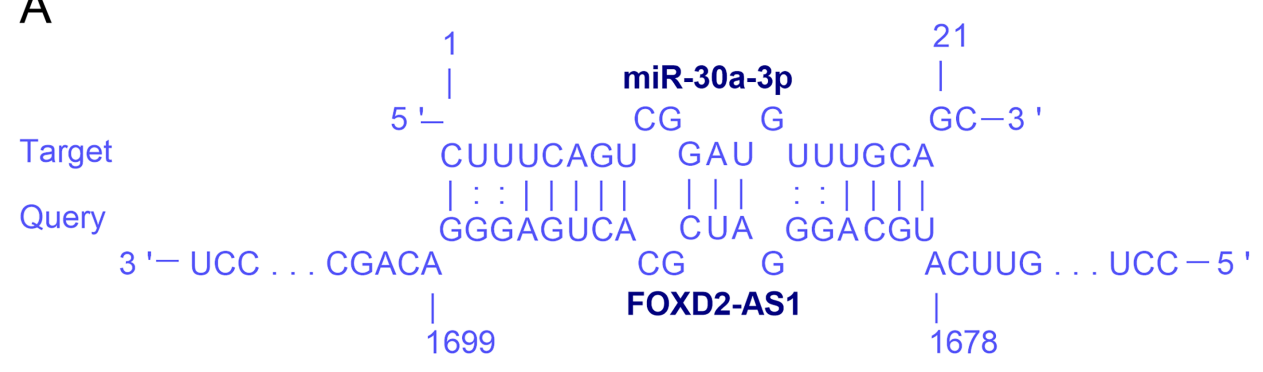

B
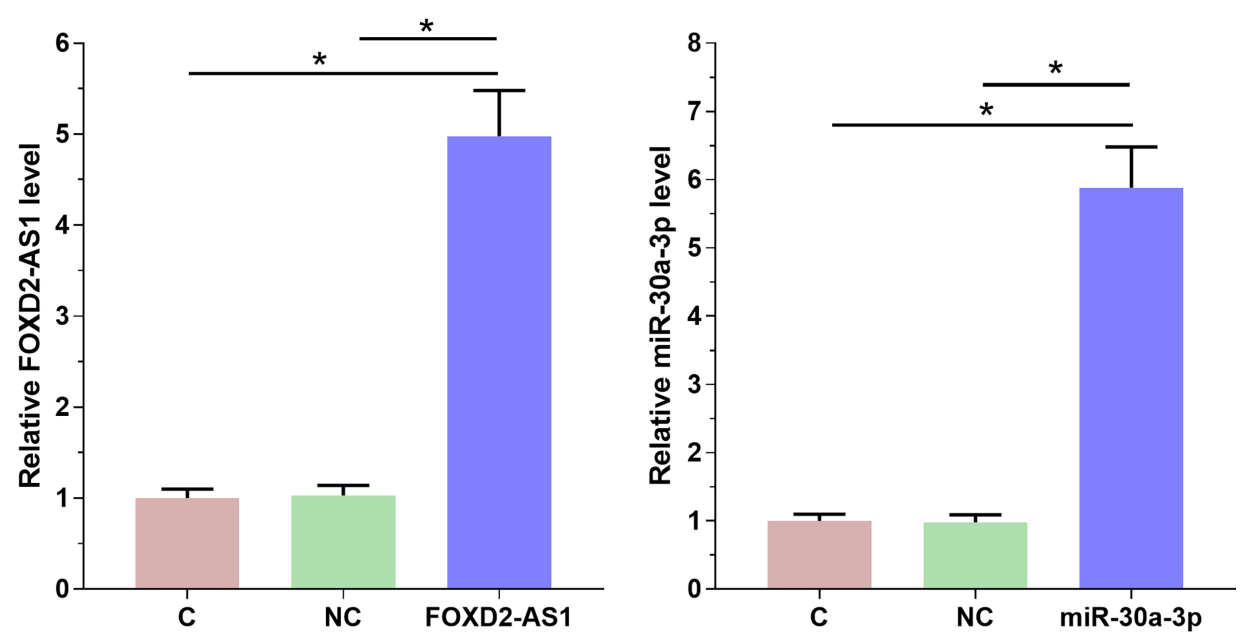

C
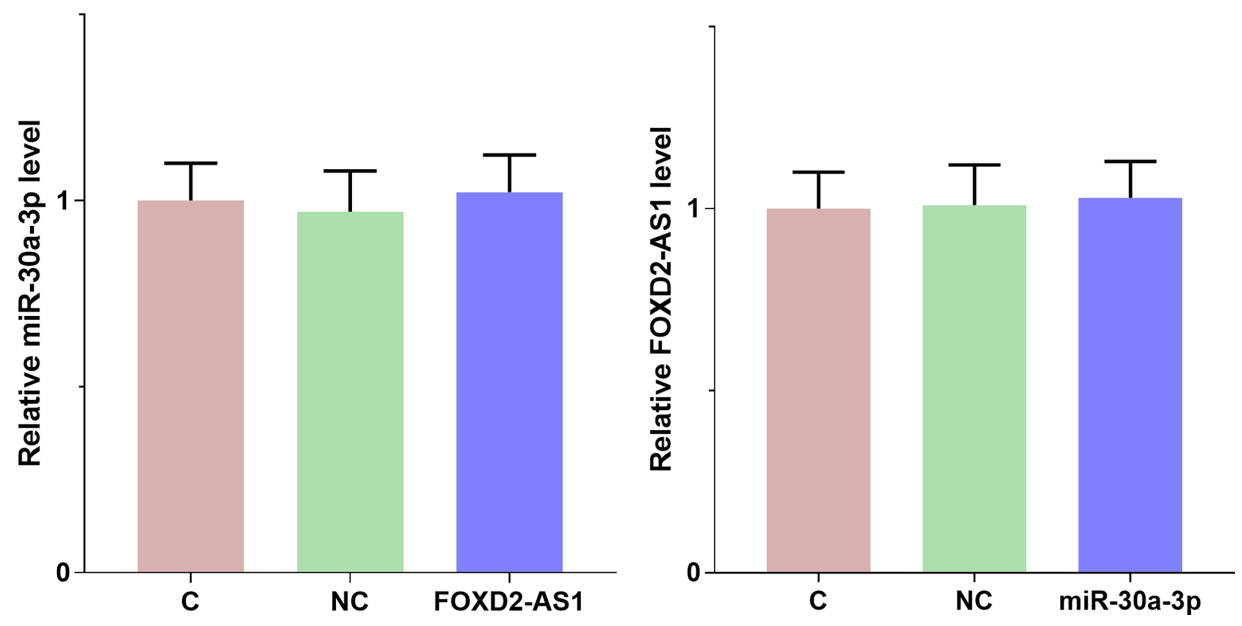

FIG. 2: MiR-30a-3p might bind to FOXD2-AS1 but did not affect its expression. The interaction between FOXD2-AS1 and miR-30a-3p was predicted by IntaRNA (http://rna.informatik.uni-freiburg.de/IntaRNA/Input.jsp). It was observed that miR-30a-3p might bind to FOXD2-AS1 (A). FOXD2-AS1 expression vector and miR-30a-3p mimic were transfected into Capan-2 cells to further investigate the interactions between FOXD2-AS1 and miR-30a-3p. Overexpression of FOXD2-AS1 and miR-30a-3p was confirmed by qPCR (B). The interaction between FOXD2-AS1 and miR-30a-3p was also analyzed by qPCR (C). Experiments were repeated three times, and mean values were presented. C, control cells without transfection; $\mathrm{NC}$, negative control cells transfected with empty vector or NC miRNA. ${ }^{*} p<0.05$. 

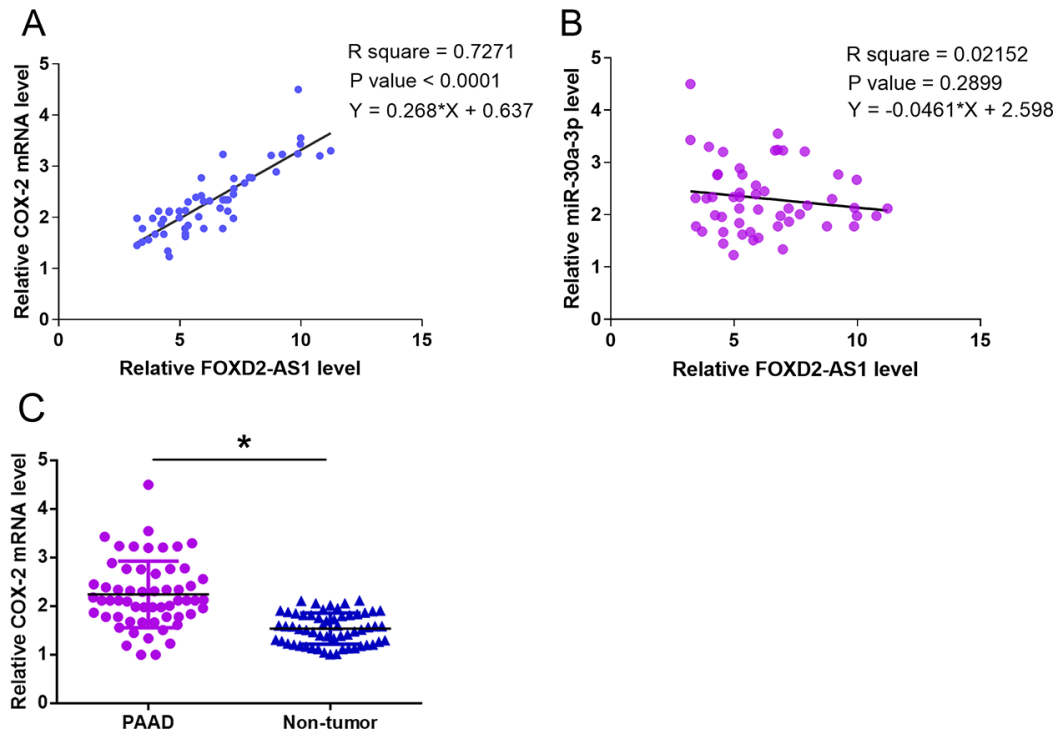

FIG. 3: FOXD2-AS1 levels were correlated with COX-2 mRNA level but not miR-30a-3p level in PAAD tissues. The expression levels of miR-30a-3p and COX-2 (a target of miR-30a-3p) mRNA were measured by qPCR. The correlations between FOXD2-AS1 COX-2 mRNA (A)/miR-30a-3p (B) were analyzed by Pearson's correlation coefficient. COX-2 mRNA levels were also compared between PAAD and non-tumor tissues $(\mathrm{C}) .{ }^{*} p<0.05$.

cancer and regulates cancer development through the Wnt/ $\beta$-catenin signaling. ${ }^{11}$ Besides, FOXD2-AS1 also interacts with miRNAs to promote nasopharyngeal carcinoma and bladder cancer progression. ${ }^{15,16}$ We showed that FOXD2-AS1 was upregulated in PAAD patients, consistent with the TCGA dataset. In addition, FOXD2-AS1 overexpression increased PAAD cell invasion and migration. Moreover, high FOXD2-AS1 levels predicted poor survival. Therefore, FOXD2-AS1 may also play an oncogenic role in PAAD by promoting tumor metastasis, leading to poor survival of PAAD patients. However, the in vivo function of FOXD2-AS1 in PAAD remains to be further explored in animal models.

MiR-30a-3p suppresses gastric cancer and liver cancer. $^{12,16}$ MiR-30a-3p targets COX-2 in gastric cancer to inhibit cell invasion and migration. ${ }^{12} \mathrm{We}$ showed that miR-30a-3p overexpression downregulated COX-2 in PAAD cells and reduced PAAD cell invasion and migration. Therefore, miR-30a-3p may also target COX-2 to regulate cancer cell invasion and migration of PAAD cells. We also found that miR-30a-3p could bind to FOXD2-AS1. However, overexpression experiments showed that FOXD2-AS1 expression was not downregulated by
miR-30a-3p. Therefore, FOXD2-AS1 is unlikely a target of miR-30a-3p. It is well established that besides regulating gene expression at multiple levels, IncRNAs may also play their roles in diverse physiological and pathological processes by serving as endogenous competing RNAs for miRNAs to suppress their roles without affecting their expression. . $^{8,918,19}$ In the present study, FOXD2-AS1 overexpression suppressed the role of miR-30a-3p in downregulating COX-2 in PAAD cells. Therefore, FOXD2-AS1 may sponge miR-30a-3p to upregulate COX-2.

\section{CONCLUSION}

FOXD2-AS1 was upregulated in PAAD and might sponge miR-30a-3p to upregulate COX-2, which in turn promote cancer cell invasion and migration.

\section{STATEMENT OF ETHICS}

Ethical clearance was obtained through the Ethics Review Committee, Zhongshan People's Hospital. Informed consent was obtained from all participants. Data collected from participants were kept confidential and were accessible only to the researchers. 

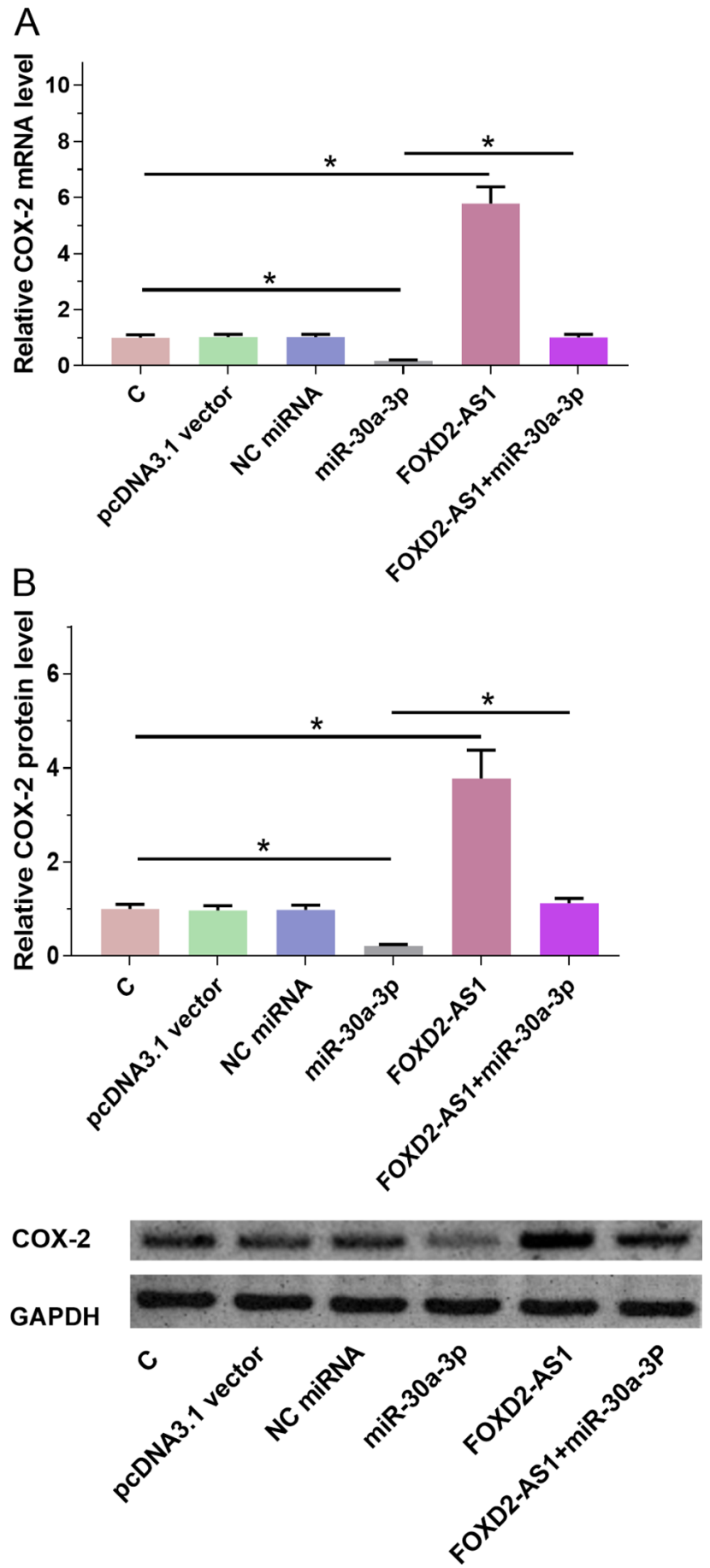

FIG 4: FOXD2-AS1 overexpression upregulated COX-2 via miR-30a-3p. QPCR and western blot were performed to analyze the effects of FOXD2-AS1 and miR-30a-3p overexpression on COX-2 expression at both mRNA (A) and protein (B) levels. Experiments were repeated three times, and mean values were presented. ${ }^{*} p<0.05$. 

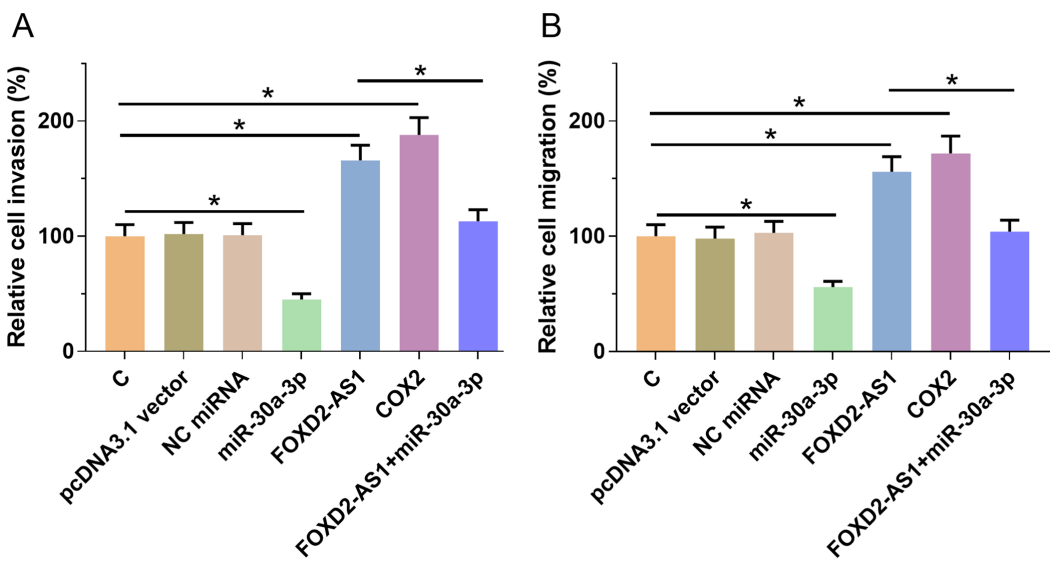

FIG. 5: FOXD2-AS1 overexpression increased Capan-2 cell invasion and migration rates via miR-30a-3p and COX2. Transwell assays were performed to analyze the effects of transfections on the invasion (A) and migration (B) of Capan-2 cells. Experiments were repeated three times, and mean values were presented. ${ }^{*} p<0.05$.

\section{FUNDING}

This work was supported by the National Natural Science Foundation of China Youth Project (Grant No. 81903029).

\section{REFERENCES}

1. Kamisawa T, Wood LD, Itoi T, Takaori K. Pancreatic cancer. Lancet. 2016;388(10039):73-85.

2. Bray F, Ferlay J, Soerjomataram I, Siegel RL, Torre LA, Jemal A. Global cancer statistics 2018: GLOBOCAN estimates of incidence and mortality worldwide for 36 cancers in 185 countries. CA Cancer J Clin. 2018;68(6):394-424.

3. Ryan DP, Hong TS, Bardeesy N. Pancreatic adenocarcinoma. N Engl J Med. 2014;371(11):1039-49.

4. Ilic M, Ilic I. Epidemiology of pancreatic cancer. World J Gastroenterol. 2016;22(44):9694-705.

5. Tanaka S. Molecular pathogenesis and targeted therapy of pancreatic cancer. Ann Surg Oncol. 2016;23(2):197-205.

6. Esteller M. Non-coding RNAs in human disease. Nat Rev Genet. 2011;12(12):861-74.

7. Taft RJ, Pang KC, Mercer TR, Dinger M, Mattick JS. Non-coding RNAs: Regulators of disease. J Pathol. 2010;220(2):126-39.

8. Parikshak NN, Swarup V, Belgard TG, Irimia M, Ramaswami G, Gandal MJ, Hartl C, Leppa V, Ubieta LT, Huang J, Lowe JK, Blencowe BJ, Horvath S, Geschwind DH. Genome-wide changes in lncRNA, splicing, and regional gene expression patterns in autism. Nature. 2016;540(7633):423-7.

9. Vidigal J A, Ventura A. The biological functions of
miRNAs: Lessons from in vivo studies. Trends Cell Biol. 2015;25(3):137-47.

10. Xu K, Zhang Z, Qian J, Wang S, Yin S, Xie H, Zhou L, Zheng S. LncRNA FOXD2-AS1 plays an oncogenic role in hepatocellular carcinoma through epigenetically silencing CDKN1B (p27) via EZH2. Exp Cell Res. 2019;380(2):198-204.

11. Rong L, Zhao R, Lu J. Highly expressed long non-coding RNA FOXD2-AS1 promotes non-small cell lung cancer progression via $\mathrm{Wnt} / \beta$-catenin signaling. Biochem Biophys Res Commun. 2017;484(3):586-91.

12. Liu X, Ji Q, Zhang C, Liu X, Liu Y, Liu N, Sui H, Zhou L, Wang S, Li Q. miR-30a acts as a tumor suppressor by double-targeting COX-2 and BCL9 in H. pylori gastric cancer models. Sci Rep. 2017;7(1):7113.

13. Livak KJ, Schmittgen TD. Analysis of relative gene expression data using real-time quantitative PCR and the 2$\triangle \Delta C T$ method. Methods. 2001;25(4):402-8.

14. Tang Z, Li C, Kang B, Gao G, Li C, Zhang Z. GEPIA: A web server for cancer and normal gene expression profiling and interactive analyses. Nucleic Acids Res. 2017;45(W1):W98-W102.

15. Chen G, Sun W, Hua X, Zeng W, Yang L. Long non-coding RNA FOXD2-AS1 aggravates nasopharyngeal carcinoma carcinogenesis by modulating miR-363-5p/S100A1 pathway. Gene. 2018;645:76-84.

16. An Q, Zhou L, Xu N. Long noncoding RNA FOXD2-AS1 accelerates the gemcitabine-resistance of bladder cancer by sponging miR-143. Biomed Pharmacother. 2018;103:415-20.

17. Wang W, Lin H, Zhou L, Zhu Q, Gao S, Xie H, Liu Z, Xu Z, Wei J, Huang X, Zheng S. MicroRNA-30a-3p inhibits tumor proliferation, invasiveness and metastasis and is downregulated in hepatocellular carcinoma. Eur J Surg Oncol. 2014;40(11):1586-94.

18. Kallen AN, Zhou XB, Xu J, Qiao C, Ma J, Yan L, Lu L, 
Liu C, Yi JS, Zhang H, Min W, Bennett AM, Gregory RI, Ding Y, Huang Y. The imprinted H19 lncRNA antagonizes let-7 microRNAs. Mol Cell. 2013;52(1):101-12.

19. Liang WC, Fu WM, Wong $\mathrm{CW}$, Wang $\mathrm{Y}$, Wang WM, Hu
GX, Zhang L, Xiao LJ, Wan DC, Zhang JF, Waye MM. The lncRNA H19 promotes epithelial to mesenchymal transition by functioning as miRNA sponges in colorectal cancer. Oncotarget. 2015;6(26):22513-25. 
